\title{
Ferroelectric Properties of a Material made of Titanium Oxide.
}

\author{
L. NrColini
}

Istituto di (himica Industriale ed Applicata, Facoltà di Ingegneria dell' Università - Bologna

$$
\text { (Nuovo Oimento, 13, } 257(1959))
$$

The figures 1 and 3 of this paper have been inadvertently inverted.

\section{Some Remarks on the Calculation of the Polarization Effects - I.}

\author{
MI. Cakrassi and G. Passatore \\ Istituto di Fisica dell' Università - Genova \\ Istituto Nazionale di Fisica Nucleare - Sezione di Genow \\ (Nuovo (imento, 13,944(1959))
}

Unfortunately we have to communicate a mistake which has been done in rewriting the 8 transition amplitudes of formula (13), p. 941:

$I^{1 \beta \gamma^{\prime} \delta}$ has to he substituted by $H^{2 \beta r}$, and conversely $I I^{2 / \gamma \delta}$ by $I^{1 \beta^{\prime 2}, s}$.

This amounts to interchange, in the same order, the values of the amplitudes on the right hand side of the first 4 equations (13) with the ones on the right hand side of the last 4 . 